\title{
Adherence to tobramycin inhaled powder vs inhaled solution in patients with cystic fibrosis: analysis of US insurance claims data
}

This article was published in the following Dove Press journal:

Patient Preference and Adherence

27 April 2017

Number of times this article has been viewed

\section{Kamal Hamed' \\ Valentino Conti \\ Hengfeng Tian' \\ Emil Loefroth ${ }^{3}$}

'Novartis Pharmaceuticals Corporation, East Hanover, NJ, USA; ${ }^{2}$ Novartis Global Service Center, Dublin, Ireland; ${ }^{3}$ Novartis Sverige AB, Täby, Sweden
Correspondence: Kamal Hamed Novartis Pharmaceuticals Corporation, One Health Plaza, East Hanover, NJ 07936, USA

Tel +l 8627784780

Email kamal.hamed@novartis.com
Purpose: Tobramycin inhalation powder (TIP), the first dry-powder inhaled antibiotic for pulmonary Pseudomonas aeruginosa infection, is associated with reduced treatment burden, increased patient satisfaction, and higher self-reported adherence for cystic fibrosis (CF) patients. We compared adherence in CF patients newly treated with TIP with those newly treated with the traditional tobramycin inhalation solution (TIS), using US insurance claims data.

Patients and methods: From the Truven MarketScan ${ }^{\circledR}$ database, we identified CF patients chronically infected with $P$. aeruginos $a$ who had been prescribed TIP between May 1, 2013 to December 31, 2014, or TIS between September 1, 2010 to April 30, 2012 with at least 12 months of continuous medical and pharmacy benefits prior to and following prescription. TIP and TIS adherence levels were assessed.

Results: A total of 145 eligible patients were identified for the TIP cohort and 306 for the TIS cohort. Significant differences in age distribution ( 25.0 vs 21.9 years for TIP vs TIS, respectively, $P=0.017)$, type of health plan $(P=0.014)$, employment status $(72.4 \%$ vs $63.4 \%$ of TIP vs TIS patients in full-time employment, $P=0.008$ ), and some comorbidities were observed between the two cohorts. Although a univariate analysis found no significant differences between TIP and TIS (odds ratio [OR] 1.411, 95\% confidence interval [CI] 0.949-2.098), TIP was moderately associated with higher adherence levels compared with TIS in a multivariable analysis, once various demographic and clinical characteristics were adjusted for. These included geographic location (OR: 1.566, CI: 1.016-2.413) and certain comorbidities.

Conclusion: This study of US patient data supports previous findings that TIP is associated with better adherence compared with TIS; however, further studies will be required to fully elucidate differences in adherence between TIP and TIS.

Keywords: Adherence, convenience, inhaler, nebulizer, Pseudomonas aeruginosa, real-world evidence, tobramycin, treatment burden

\section{Introduction}

Cystic fibrosis (CF) is an autosomal recessive inherited disease, caused by mutations in the cystic fibrosis transmembrane conductance regulator gene and characterized by thick secretions, resulting in multisystem complications. ${ }^{1}$ Morbidity and mortality in CF are primarily attributed to recurrent respiratory infections and progressive obstructive lung disease, leading to overall deterioration of pulmonary function, respiratory failure, and ultimately death. ${ }^{2}$ Pseudomonas aeruginosa represents the archetypal CF pathogen, causing chronic infection in $\sim 60 \%-75 \%$ of adults with $\mathrm{CF}^{3}$ Its management is notoriously difficult because of its extensive multidrug resistance. ${ }^{4,5}$ 
The expanded use of antipseudomonal inhaled antibiotics, including traditional nebulized tobramycin inhalation solution (TIS), has contributed greatly to improvements in CF management and patient outcomes. ${ }^{6-9}$ However, treatment regimens and drug delivery methods for inhaled antibiotics can be complex and represent a substantial treatment burden; ${ }^{10,11}$ thus, poor adherence is often reported. Poor adherence is, in turn, associated with worse patient outcomes and higher health care utilization and costs. ${ }^{12,13}$ Tobramycin inhalation powder (TIP; TOBI ${ }^{\circledR}$ Podhaler ${ }^{\mathrm{TM}}$, Novartis Pharma AG, Basel, Switzerland) is the first dry-powder inhaled antibiotic available for use in CF patients; it uses PulmoSphere ${ }^{\circledR}$ technology for efficient drug delivery into the lower airways. ${ }^{14}$ TIP has been found to be equally efficacious as TIS, ${ }^{15-18}$ and was approved by the US Food and Drug Administration in 2013. Furthermore, TIP has been recognized in recent treatment guidelines. ${ }^{19}$ In addition to its equivalent efficacy, TIP is also associated with improved patient convenience and satisfaction compared with TIS. ${ }^{20}$

Whether these improvements in convenience and satisfaction translate into improved adherence has yet to be determined. Indeed, to date, only a few European studies ${ }^{21,22}$ have assessed adherence rates for TIP compared with TIS in a real-world setting. However, these studies used self-reported measures of adherence, which are subjective and can often be inflated. ${ }^{23}$ The objective of this study was, therefore, to compare adherence in $\mathrm{CF}$ patients chronically infected with $P$. aeruginosa and newly treated with either TIP or TIS, using commercial US claims data.

\section{Materials and methods}

\section{Data source and patient record selection}

This retrospective cohort study analyzed Truven Health Analytics data from the MarketScan database, ${ }^{24}$ covering patient data from the period September 1, 2009 to December 31, 2015. In the database, medical claims, outpatient prescription drug claims, and person-level enrollment data are linked with unique patient identifiers. All study data were accessed using techniques compliant with the Health Insurance Portability and Accountability Act of 1996. No identifiable protected health information was extracted during the study, and hence, this study did not require informed consent or institutional review board approval.

Patients with CF chronically infected with $P$. aeruginosa were eligible for this study. Patients with CF were considered chronically infected with $P$. aeruginosa if they had $\geq 2$ claims of TIP/TIS during the 12-month follow-up period. Patients were identified through May 1, 2013 to December 31, 2014 for the cohort of newly treated TIP patients and through
September 1, 2010 to April 30, 2012 for the cohort of newly treated TIS patients. The start date of the identification period for TIP patients was selected based upon the date TIP was first dispensed postapproval, and the end date was selected to ensure that all patient data had a 12-month follow-up period. The identification period for TIS patients was selected to enable a 12-month follow-up period where TIS-to-TIP switching was not a possibility. The first prescriptions of TIP or TIS during these identification periods were designated as their index date.

All patients were required to have at least one claim for TIP or TIS during the identification period and at least one claim for CF (ICD-9-CM: 277.0x or ICD-10-CM: E84.x), as well as eligibility for continuous medical and pharmacy benefits during both the pre-index period (at least 12 months enrollment before the index date) and the post-index period (at least 12 months enrollment after and including the index date). Patients who underwent lung transplantation during the pre-index and post-index period were excluded from this study. Other exclusion criteria included any claim for TIS or TIP during the pre-index period.

\section{Endpoints}

The primary endpoint of this study was comparing 12-month adherence to TIP with 12-month adherence to TIS, with adherence measured as a dichotomous variable; high vs low/medium utilization. High utilization was defined as $\geq 4$ cycles, low/medium utilization were defined as $\leq 2$ and 3 cycles, respectively. The secondary endpoint was to compare adherence measured as a continuous outcome (mean number of cycles). The measures of adherence were defined according to the cycles of therapy during the 12-month follow-up, where a 56-day supply was assigned to each claim and considered as 1 cycle of therapy. This reflects the unique administration schedule of TIP/TIS therapy, where each claim encompasses 28 days on therapy and 28 days off.

\section{Sociodemographic and clinical characteristics}

Patient demographic characteristics were captured at the index date and clinical variables were measured during the pre-index period, such as the Charlson Comorbidity Index (CCI), single items of the CCI, and other selected comorbidities of relevance to CF patients, ${ }^{25-27}$ defined by $\geq 1$ inpatient/ outpatient diagnosis or procedure.

\section{Statistical analyses}

Baseline characteristics, covariates, and study outcomes were summarized descriptively; categorical data were presented 
as counts and proportions, and summary statistics were presented for continuous data. For the primary objective (12-month adherence to TIS compared with that to TIP), unadjusted and adjusted odds ratios (ORs) of being highly adherent, relative to low/medium adherence as reference, were estimated using logistic regression. For the secondary objective, unadjusted and adjusted ratios of mean number of TIP cycles to mean number of TIS cycles were estimated using a negative binomial regression model. Ninety-five percent confidence intervals (CIs) were reported for both the univariate and multivariable analyses.

Based on a feasibility analysis of Dutch pharmacy-based data (data on file), to detect a significant between-group difference in adherence assuming an adherence rate of 0.40 for the TIP group and of 0.22 for the TIS group, at a level of $P<0.05$ with a power of $80 \%$, a total of 204 patients (102 per group) were required. A mixed approach for the covariate selection in the multivariable model was used, with age as a categorical variable, and gender, CCI, depression, and anxiety included as a priori independent variables; other variables were only included if associated with both outcome and exposure. A cloud-based Linux x64 SAS 9.4 program was used for all analyses.

\section{Results}

\section{Patient identification and characteristics}

A total of 145 eligible patients were identified for the cohort of newly treated TIP patients and 306 for the cohort of newly treated TIS patients (Figure 1). There were significant differences in the age distribution (mean age, 25.0 years for the TIP population vs 21.9 years for the TIS population, $P=0.017)$, type of health plan $(P=0.014)$, and employment status $(72.4 \%$ of TIP patients in active full-time employment vs $63.4 \%$ of TIS patients, $P=0.008)$. Among the comorbidities included in the CCI, diabetes without chronic complication was significantly different (33.8\% for TIP and $20.9 \%$ for TIS, $P=0.003$ ). From the selected comorbidities for this study, three were significantly different: pancreatic insufficiency (93.8\% for TIP and $86.9 \%$ for TIS, $P=0.018$ ), malnutrition (30.3\% for TIP and $19.0 \%$ for TIS, $P=0.007$ ), and chronic sinusitis (64.1\% for TIP and $52.3 \%$ for TIS, $P=0.019)$. All other demographic characteristics (Table 1) and clinical variables (Table 2) were similar across the two populations.

\section{Twelve-month adherence in CF patients newly treated with TIP compared with TIS}

For the primary objective, although a tendency toward greater adherence to TIP compared with TIS was observed, univariate analysis found no statistically significant differences between TIP and TIS (OR: 1.411, 95\% CI: 0.949-2.098). In the multivariable analysis, there was no significant effect on adherence when comparing TIP with TIS while adjusting for the base case variables: gender, age, CCI, and depression (OR: 1.521, 95\% CI: 0.992-2.333; Table 3), nor when adding employment status, health plan, or co-pay to the model. However, when geographic location was included in the multivariable model as a proxy for variables related to adherence, ${ }^{28}$ a significant effect was observed (OR: 1.566, 95\% CI: 1.016-2.413; Table 3). Pancreatic insufficiency, gastroesophageal reflux, liver and lung transplant and aftercare, and anxiety all resulted in significant effects on adherence to TIP compared with TIS after selected comorbidities were added to the analysis (Table 3), implying that adherence to TIP or TIS may have been affected by those comorbidities.

When comparing TIS and TIP adherence measured as a continuous variable for the secondary objective, no significant differences were observed between the mean number of treatment cycles in the TIP cohort compared with the TIS cohort (3.85 for TIP and 3.65 for TIS, $P=0.308$ ).

\section{Discussion}

The aim of our study was to compare adherence for the two tobramycin delivery routes. The adherence rates reported in this study (3.85 cycles and 3.65 cycles during the first year of treatment with TIP and TIS, respectively, out of a maximum of 6 cycles per year) are equivalent to a medical possession ratio $\geq 60 \%$. These are in accordance with other studies that have used either data from the MarketScan database to assess adherence with inhaled tobramycin $(51 \%),{ }^{12}$ or pharmacy refill data to assess adherence with combined nebulized medications, including inhaled tobramycin $(\sim 70 \%){ }^{23}$ Briesacher et al have previously reported much lower adherence with TIS, as only $6 \%$ of patients fell under the high utilization category ( $\geq 4$ cycles per year). ${ }^{13}$ Early dropout patients were not excluded in that study, which may have led to lower rates of adherence compared with our study, in which early dropout patients were excluded to ensure that all patient data were from patients chronically infected with $P$. aeruginosa. Thus, in our study, we aimed to provide further evidence regarding 12-month adherence to TIP compared with TIS using insurance claims data in a larger cohort of patients chronically infected with $P$. aeruginosa and newly treated with these medications. From the crude analysis for the primary and secondary objectives, no difference was observed in adherence between TIP and TIS treatment. However, in a multivariable analysis adjusted for demographic variables 


\begin{tabular}{|c|c|}
\hline \multicolumn{2}{|c|}{ Include at least one claim for TIP or TIS during the identification period } \\
\hline TIP: $N=1,194$ & TIS: $N=3,608$ \\
\hline \multicolumn{2}{|c|}{ Include at least one claim for cystic fibrosis ${ }^{a}$ in the pre-index or post-index period (including index date) } \\
\hline TIP: $N=1,111$ & TIS: $N=2,217$ \\
\hline \multicolumn{2}{|c|}{$\begin{array}{l}\text { Include continuous medical and pharmacy benefits eligibility during } 12 \text { months pre-index } \\
\text { period (including index date) }\end{array}$} \\
\hline TIP: $N=703$ & TIS: $N=1,322$ \\
\hline \multicolumn{2}{|c|}{ Include continuous medical and pharmacy benefits eligibility during 12 months post-index period } \\
\hline TIP: $N=502$ & TIS: $N=1,008$ \\
\hline \multicolumn{2}{|c|}{ Exclude patients with lung transplantation during pre-index and post-index period (including index date) } \\
\hline TIP: $N=499$ & TIS: $N=990$ \\
\hline \multicolumn{2}{|c|}{$\begin{array}{l}\text { Exclude patients with at least one claim for TIS or TIP during the pre-index period (excluding index date) } \\
\text { ie, the patients must be newly treated with either tobramycin drug (TIP or TIS) }\end{array}$} \\
\hline TIP: $N=237$ & TIS: $N=507$ \\
\hline \multicolumn{2}{|c|}{ Exclude patients without any further claim of drug received at index date (ie, early dropout) } \\
\hline TIP: N=145 & TIS: N=306 \\
\hline
\end{tabular}

Figure I CONSORT diagram showing patient selection for primary and secondary objectives.

Notes: ${ }^{a}$ Cystic Fibrosis ICD-9-CM: 277.0x, ICD-I0-CM: E84.x. ' ${ }^{b}$ Enrollment gap allowance of 45 days applied.

Abbreviations: CONSORT, Consolidated Standards of Reporting Trials; TIP, tobramycin inhalation powder; TIS, tobramycin inhalation solution.

and clinical characteristics, TIP was moderately associated with greater adherence levels compared with TIS.

Studies examining differences between the traditional nebulized solution and the novel dry-powder form of tobramycin in a clinical trial setting have highlighted the equivalent efficacy and safety between the two delivery methods, and have demonstrated increased patient satisfaction and convenience with TIP compared with TIS. ${ }^{16-19}$ Real-world data have also demonstrated that satisfaction with TIP is high among patients with $\mathrm{CF}$ and $P$. aeruginosa infection. ${ }^{20}$ High adherence to inhaled antibiotics has been found to be associated with patient satisfaction, ${ }^{29}$ so it is perhaps unsurprising that preliminary evidence suggests that TIP is associated with improved adherence, tolerability and decreased exacerbation rates in comparison with TIS..$^{18,19}$ Indeed, Harrison et al reported that the proportion of patients reporting "excellent adherence" to TIP was $83 \%$ at 12 months, a dramatic increase from the $43 \%$ of patients who reported "excellent adherence" with TIS at baseline before transitioning to TIP. ${ }^{21}$ Some of the limitations of the above study included a relatively small sample size and the potential for recall bias, as adherence was self-reported by the patients. ${ }^{21}$ 
Table I Summary of patient demographic characteristics

\begin{tabular}{|c|c|c|}
\hline $\begin{array}{l}\text { Baseline characteristics and } \\
\text { covariates }\end{array}$ & $\begin{array}{l}\text { Tobramycin inhalation } \\
\text { powder cohort }(\mathrm{N}=145)\end{array}$ & $\begin{array}{l}\text { Tobramycin inhalation } \\
\text { solution cohort }(\mathrm{N}=306)\end{array}$ \\
\hline Age, mean (SD) & $25.0(\mathrm{II} . \mathrm{I})$ & $21.9(16.2)$ \\
\hline \multicolumn{3}{|l|}{ Age group, years n (\%) } \\
\hline$<6$ & $0(0.0)$ & $33(10.8)$ \\
\hline$\geq 6$ and $<12$ & $2(1.4)$ & $49(16.0)$ \\
\hline$\geq 12$ and $<18$ & $39(26.9)$ & $65(21.2)$ \\
\hline$\geq 18$ and $<25$ & $49(33.8)$ & $70(22.9)$ \\
\hline$\geq 25$ and $<35$ & $33(22.8)$ & $36(11.8)$ \\
\hline$\geq 35$ & $22(15.2)$ & $53(17.3)$ \\
\hline \multicolumn{3}{|l|}{ Gender, n (\%) } \\
\hline Male & $80(55.2)$ & $154(50.3)$ \\
\hline Female & $65(44.8)$ & $152(49.7)$ \\
\hline \multicolumn{3}{|l|}{ Employment status, n (\%) } \\
\hline Active full time & $105(72.4)$ & $194(63.4)$ \\
\hline Other/unknown & $40(27.6)$ & $112(36.6)$ \\
\hline \multicolumn{3}{|l|}{ Health care plan type, n (\%) } \\
\hline Basic/major medical & $0(0.0)$ & $0(0.0)$ \\
\hline Comprehensive & $2(1.4)$ & $7(2.3)$ \\
\hline Exclusive provider organization & $\mathrm{I}(0.7)$ & $7(2.3)$ \\
\hline Health maintenance organization & $18(12.4)$ & $57(18.6)$ \\
\hline POS & $16(11.0)$ & $22(7.2)$ \\
\hline Preferred provider organization & $77(53.1)$ & $177(57.8)$ \\
\hline POS with capitation & I (0.7) & $5(1.6)$ \\
\hline Consumer-driven health plan & $17(11.7)$ & $17(5.6)$ \\
\hline High-deductible health plan & II (7.6) & $8(2.6)$ \\
\hline Missing & $2(1.4)$ & $6(2.0)$ \\
\hline \multicolumn{3}{|l|}{ Geographic location (US), n (\%) } \\
\hline Northeast & $30(20.7)$ & $43(14.1)$ \\
\hline North central & $39(26.9)$ & $76(24.8)$ \\
\hline South & $52(35.9)$ & $112(36.6)$ \\
\hline West & $23(15.9)$ & $73(23.9)$ \\
\hline Unknown & $\mathrm{I}(0.7)$ & $2(0.7)$ \\
\hline $\begin{array}{l}\text { Co-payment (of index treatment), } \\
\text { mean US\$ (SD) }\end{array}$ & $60.3(83.8)$ & $49.0(96.1)$ \\
\hline
\end{tabular}

Abbreviations: POS, point of service; SD, standard deviation.

A number of patient demographic and clinical characteristics were found to vary between the two different populations in the pre-index period and at index date. TIP users, for example, were more likely to be older and in active full-time employment. Treatment complexity in $\mathrm{CF}$ has been reported to be the highest among older patients, ${ }^{30}$ and administration times are significantly shorter for TIP (5.6 vs 19.7 minutes for TIP vs TIS, respectively), ${ }^{13}$ so it may be the case that older patients who already have complex treatment regimens and a busy work schedule have a preference for TIP rather than TIS. In another study, all of the patients questioned $(n=35)$ expressed a preference for TIP over a nebulized antibiotic with regard to its shorter administration time, providing further support toward the concept that patients who require a less time-consuming therapy may prefer TIP. ${ }^{20}$
Differences in patient demographic and clinical characteristics could influence adherence rates independent of delivery methods between the two populations. For example, Quittner et al have previously demonstrated that age is associated with treatment adherence in $\mathrm{CF}$, with younger patients more likely to be adherent than their older counterparts, ${ }^{12}$ which may be due to the benefits of parental supervision in younger patients. To establish if any differences in patient characteristics were independently influencing adherence and thus affecting the results of the crude analysis, we conducted a multivariable analysis, adjusting for any potentially confounding variables.

Although age and employment status did significantly differ between the TIP and TIS populations, no significant differences between treatment adherence to TIP compared with TIS were observed when these were adjusted for in the 
Table 2 Summary of patient clinical characteristics

\begin{tabular}{|c|c|c|}
\hline Pre-index comorbidities & $\begin{array}{l}\text { Tobramycin inhalation } \\
\text { powder cohort }(\mathrm{N}=\mid 45)\end{array}$ & $\begin{array}{l}\text { Tobramycin inhalation } \\
\text { solution cohort }(\mathrm{N}=306)\end{array}$ \\
\hline $\mathrm{CCl}$, mean (standard deviation) & $2.44(1.92)$ & $2.35(2.07)$ \\
\hline \multicolumn{3}{|l|}{ Single items of $\mathrm{CCl}, \mathrm{n}(\%)$} \\
\hline Myocardial infarction & $0(0.0)$ & $3(1.0)$ \\
\hline Congestive heart failure & $14(9.7)$ & $26(8.5)$ \\
\hline Peripheral vascular disease & $I(0.7)$ & $8(2.6)$ \\
\hline Cerebrovascular disease & $14(9.7)$ & $37(12.1)$ \\
\hline Dementia & $0(0.0)$ & $0(0.0)$ \\
\hline Chronic pulmonary disease & $12 \mid(83.5)$ & $245(80.1)$ \\
\hline Rheumatic disease & $86(59.3)$ & $176(57.5)$ \\
\hline Peptic ulcer disease & $3(2.1)$ & $3(1.0)$ \\
\hline Mild liver disease & $13(9.0)$ & $31(10.1)$ \\
\hline Diabetes without chronic complication & $49(33.8)$ & $64(20.9)$ \\
\hline Diabetes with chronic complication & $9(6.2)$ & $10(3.3)$ \\
\hline Hemiplegia or paraplegia & I (0.7) & $8(2.6)$ \\
\hline Renal disease & $5(3.5)$ & $14(4.6)$ \\
\hline Any malignancy & $4(2.8)$ & $14(4.6)$ \\
\hline Moderate or severe liver disease & $3(2.1)$ & $5(1.6)$ \\
\hline Metastatic solid tumor & $0(0.0)$ & $3(1.0)$ \\
\hline Acquired immune deficiency syndrome/ & $\mathrm{I}(0.7)$ & $0(0.0)$ \\
\hline human immunodeficiency virus & & \\
\hline \multicolumn{3}{|l|}{ Selected comorbidities, n (\%) } \\
\hline Pancreatic insufficiency & $136(93.8)$ & $266(86.9)$ \\
\hline Gastroesophageal reflux & $48(33.1)$ & $103(33.7)$ \\
\hline Chronic sinusitis & $93(64.1)$ & $160(52.3)$ \\
\hline Malnutrition & $44(30.3)$ & $58(19.0)$ \\
\hline Osteoporosis & $21(14.5)$ & $32(10.5)$ \\
\hline Liver and lung transplant and aftercare & $8(5.5)$ & $9(2.9)$ \\
\hline Depression & $33(22.8)$ & $38(12.4)$ \\
\hline Anxiety & $6(4.1)$ & $5(1.6)$ \\
\hline
\end{tabular}

Abbreviation: $\mathrm{CCl}$, Charlson Comorbidity Index.

multivariable analysis, although there was a trend toward higher adherence with TIP relative to TIS. A Phase 4 study assessing self-reported adherence to TIP, as measured by the Morisky score, found higher rates of adherence in children and teenagers compared with adults. ${ }^{22}$ The fact that we did not find

Table 3 Multivariable analysis of adherence to tobramycin inhalation powder relative to tobramycin inhalation solution (high vs low/medium utilization)

\begin{tabular}{ll}
\hline Variables included in the model & $\begin{array}{l}\text { Odds ratio } \\
\text { estimate }(95 \% \\
\text { confidence interval) }\end{array}$ \\
\hline Index drug, gender, age, Charlson Comorbidity & $\mathrm{I} .52 \mathrm{I}(0.992-2.333)$ \\
Index, depression (a priori variables) & $\mathrm{I} .530(0.996-2.35 \mathrm{I})$ \\
A priori variables + employment status & $\mathrm{I} .448(0.933-2.248)$ \\
A priori variables + health plan type & $\mathrm{I} .566(\mathrm{I} .016-2.4 \mathrm{I} 3)$ \\
A priori variables + geographic location & $\mathrm{I} .517(0.989-2.326)$ \\
A priori variables + co-payment & \\
A priori variables + comorbidities & $\mathrm{I} .555(\mathrm{I} .0 \mathrm{I} 2-2.390)$ \\
$\quad$ Pancreatic insufficiency & $\mathrm{I} .549(\mathrm{I} .007-2.383)$ \\
Gastroesophageal reflux & $\mathrm{I} .449(0.976-2.30 \mathrm{I})$ \\
Chronic sinusitis & $\mathrm{I} .530(0.996-2.350)$ \\
Malnutrition & $\mathrm{I} .519(0.990-2.330)$ \\
Osteoporosis & $\mathrm{I} .535(\mathrm{I} .000-2.356)$ \\
Liver and lung transplant and aftercare & $\mathrm{I} .540(\mathrm{I} .003-2.365)$ \\
\hline Anxiety &
\end{tabular}

age to significantly impact adherence may lie in the use of different adherence measures between the two studies; however, we cannot rule out the possibility that age influenced adherence across both patient populations in our study but the effect was not found to be significant because of sample size.

Geographic location is a variable that has been found in the literature to affect adherence and serves as a proxy for other variables related to adherence such as health literacy and care access. ${ }^{28}$ Indeed, when geographic location was adjusted for in our multivariable analysis, TIP was found to be associated with higher utilization relative to TIS. This was also the case when certain comorbidities were adjusted for, although this should be interpreted with caution because of the significant differences in some of the comorbidities, but not in others. For example, when anxiety was adjusted for, TIP was found to be associated with higher utilization relative to TIS, but this was not the case when depression was adjusted for. One could interpret from this that anxiety, but not depression, has a significant impact on treatment adherence to TIP and TIS, which may be surprising as depression has previously been found to be associated with noncompliance to treatments in patients with $\mathrm{CF} .{ }^{31}$ However, as a general trend toward higher adherence with TIP relative to TIS was observed when 
all the variables were adjusted for, it may be that a larger sample size is required to detect these differences.

Overall, these results are promising and offer further support to previous findings that patients treated with TIP demonstrate better adherence compared with TIS. Further studies will be required to fully elucidate differences in adherence between the two treatment methods.

\section{Limitations}

Health care claims data are subject to several limitations, such as potentially different coding patterns, and inaccuracies in coding and data entry. Additionally, in the post-index period where medications may be obtained from a pharmacy setting, it cannot be known whether medications are being taken by the patients as prescribed. For a claims data study, there is the clear limitation of selection bias, which was addressed in this study by selecting newly treated patients with TIP or TIS and through adjusting the estimates for the covariates associated with TIP or TIS and adherence. We chose not to create two equally balanced groups to avoid reductions in sample size, and we included only naïve TIP and TIS patients to generate more generalizable results. Imbalances between the two groups do not seem to have played a major role in determining adherence, as shown in Table 3: all the multivariable models produced very similar results. Data regarding the causality or reasons for switching to or stopping TIP were not collected in this study; such data may have provided further insight into why adherence may be similar across the two groups in the univariate analysis. It should also be noted that these data were based on medical care delivered through health insurance plans, rather than the general population.

\section{Conclusions}

The study adds support to the concept that TIP is associated with better adherence compared with TIS. This could be due to the similar efficacy but shorter administration time, ease of use, and patient overall satisfaction with this system of delivery.

\section{Acknowledgments}

Editorial assistance in the preparation of this manuscript was provided by Antonia Bowman of Seren Communications, an Ashfield Company, part of UDG Healthcare, the funding for which was provided by Novartis Pharma AG.

\section{Disclosure}

$\mathrm{KH}$ and HT are employees of Novartis Pharmaceuticals Corporation, East Hanover, NJ, USA. VC is an employee of Novartis Global Service Center, Dublin, Ireland. EL is an employee of Novartis Sverige AB, Täby, Sweden. The authors report no other conflicts of interest in this work.

\section{References}

1. Knowles MR, Durie PR. What is cystic fibrosis? N Engl J Med. 2002; 347(6):439-442.

2. Flume PA. Pulmonary complications of cystic fibrosis. Respir Care. 2009;54(5):618-627.

3. Cystic Fibrosis Foundation Patient Registry. 2015 Annual Data Report. Bethesda, MD: Cystic Fibrosis Foundation; 2016.

4. Potron A, Poirel L, Nordmann P. Emerging broad-spectrum resistance in Pseudomonas aeruginosa and Acinetobacter baumannii: mechanisms and epidemiology. Int J Antimicrob Agents. 2015;45(6): 568-585.

5. Poole K. Pseudomonas aeruginosa: resistance to the max. Front Microbiol. 2011;2:65.

6. McCoy KS, Quittner AL, Oermann CM, Gibson RL, RetschBogart GZ, Montgomery AB. Inhaled aztreonam lysine for chronic airway Pseudomonas aeruginosa in cystic fibrosis. Am J Respir Crit Care Med. 2008;178(9):921-928.

7. Retsch-Bogart GZ, Quittner AL, Gibson RL, et al. Efficacy and safety of inhaled aztreonam lysine for airway pseudomonas in cystic fibrosis. Chest. 2009;135(5):1223-1232.

8. Ramsey BW, Pepe MS, Quan JM, et al. Intermittent administration of inhaled tobramycin in patients with cystic fibrosis. Cystic Fibrosis Inhaled Tobramycin Study Group. N Engl J Med. 1999;340(1):23-30.

9. Ryan G, Singh M, Dwan K. Inhaled antibiotics for long-term therapy in cystic fibrosis. Cochrane Database Syst Rev. 2011;(3): CD001021.

10. Sawicki GS, Sellers DE, Robinson WM. High treatment burden in adults with cystic fibrosis: challenges to disease self-management. J Cyst Fibros. 2009;8(2):91-96.

11. Bodnár R, Mészáros A, Oláh M, Ágh T. Inhaled antibiotics for the treatment of chronic Pseudomonas aeruginosa infection in cystic fibrosis patients: challenges to treatment adherence and strategies to improve outcomes. Patient Prefer Adherence. 2016;10:183-193.

12. Quittner AL, Zhang J, Marynchenko M, et al. Pulmonary medication adherence and health-care use in cystic fibrosis. Chest. 2014;146(1): $142-151$.

13. Briesacher BA, Quittner AL, Saiman L, Sacco P, Fouayzi H, Quittell LM. Adherence with tobramycin inhaled solution and health care utilization. BMC Pulm Med. 2011;11:5.

14. Smyth AR, Bell SC, Bojcin S, et al. European cystic fibrosis society standards of care: best practice guidelines. J Cyst Fibros. 2014; 13(Suppl 1):S23-S42.

15. Geller DE, Weers J, Heuerding S. Development of an inhaled drypowder formulation of tobramycin using PulmoSphere ${ }^{\mathrm{TM}}$ technology. J Aerosol Med Pulm Drug Deliv. 2011;24(4):175-182.

16. Konstan MW, Flume PA, Kappler M, et al. Safety, efficacy and convenience of tobramycin inhalation powder in cystic fibrosis patients: the EAGER trial. J Cyst Fibros. 2011;10(1):54-61.

17. Somayaji R, Parkins MD. Tobramycin inhalation powder: an efficient and efficacious therapy for the treatment of Pseudomonas aeruginosa infection in cystic fibrosis. Ther Deliv. 2016;6(2):121-137.

18. Lam J, Vaughan S, Parkins MD. Tobramycin inhalation powder (TIP): an efficient treatment strategy for the management of chronic Pseudomonas aeruginosa infection in cystic fibrosis. Clin Med Insights Circ Respir Pulm Med. 2013;7:61-77.

19. Littlewood KJ, Higashi K, Jansen JP, et al. A network meta-analysis of the efficacy of inhaled antibiotics for chronic Pseudomonas infections in cystic fibrosis. J Cyst Fibros. 2012;11(5):419-426.

20. Greenberg J, Palmer JB, Chan WW, et al. Treatment satisfaction in cystic fibrosis: early patient experience with tobramycin inhalation powder. Patient Prefer Adherence. 2016;10:2163-2169.

21. Harrison MJ, McCarthy M, Fleming C, et al. Inhaled vs nebulised tobramycin: A real world comparison in adult cystic fibrosis (CF). J Cyst Fibros. 2014;13(6):692-698. 
22. Hamed K, Debonnett L. Tobramycin inhalation powder for the treatment of pulmonary Pseudomonas aeruginosa infection in patients with cystic fibrosis: a review based on clinical evidence. Ther Adv Respir Dis. In press 2016.

23. Modi AC, Lim CS, Yu N, Geller D, Wagner MH, Quittner AL. A multi-method assessment of treatment adherence for children with cystic fibrosis. J Cyst Fibros. 2006;5(3):177-185.

24. Danielson E. Health research data for the real world: the MarketScan Databases. White Paper. Truven Health Analytics, Ann Arbor, MI; 2014.

25. Ouyang L, Grosse SD, Amendah DD, Schechter MS. Healthcare expenditures for privately insured people with cystic fibrosis. Pediatr Pulmonol. 2009;44(10):989-996.

26. White T, Miller J, Smith GL, McMahon WM. Adherence and psychopathology in children and adolescents with cystic fibrosis. Eur Child Adolesc Psychiatry. 2009;18(2):96-104.

27. DiMatteo MR, Lepper HS, Croghan TW. Depression is a risk factor for noncompliance with medical treatment: meta-analysis of effects of anxiety and depression on patient adherence. Arch Intern Med. 2000;160(14): 2101-2107.
28. Couto JE, Panchal JM, Lal LS, et al. Geographic variation in medication adherence in commercial and Medicare part D populations. J Manag Care Spec Pharm. 2014;20(8):834-842.

29. Regnault A, Balp MM, Kulich K, Viala-Danten M. Validation of the treatment satisfaction questionnaire for medication in patients with cystic fibrosis. J Cyst Fibros. 2012;11(6):494-501.

30. Sawicki GS, Ren CL, Konstan MW, Millar SJ, Pasta DJ, Quittner AL; Investigators and Coordinators of the Epidemiologic Study of Cystic Fibrosis. Treatment complexity in cystic fibrosis: trends over time and associations with site-specific outcomes. J Cyst Fibros. 2013;12(5): $461-467$.

31. DiMatteo MR, Lepper HS, Croghan TW. Depression is a risk factor for noncompliance with medical treatment: meta-analysis of effects of anxiety and depression on patient adherence. Arch Intern Med. 2000; 160(14):2101-2107.

\section{Publish your work in this journal}

Patient Preference and Adherence is an international, peer-reviewed, open access journal that focuses on the growing importance of patient preference and adherence throughout the therapeutic continuum. Patient satisfaction, acceptability, quality of life, compliance, persistence and their role in developing new therapeutic modalities and compounds to optimize clinical outcomes for existing disease states are major areas of interest for the journal. This journal has been accepted for indexing on PubMed Central. The manuscript management system is completely online and includes a very quick and fair peer-review system, which is all easy to use. Visit http://www dovepress.com/testimonials.php to read real quotes from published authors. 